\title{
Electronic Transport in Self-organised Molecular Nanostructured Devices
}

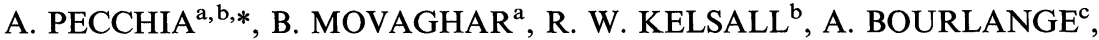 \\ S. D. EVANS ${ }^{\mathrm{a}, \mathrm{c}}$, B. J. HICKEY ${ }^{\mathrm{c}}$ and N. BODEN ${ }^{\mathrm{a}}$ \\ ${ }^{\mathrm{a}}$ Centre for Self Organising Molecular Systems, ${ }^{\mathrm{b}}$ School of Electronic and Electrical Engineering, \\ ${ }^{c}$ Department of Physics and Astronomy, The University of Leeds, Leeds, LS29JT UK
}

\begin{abstract}
We analyse the frequency dependent conductivity of a system which is fabricated using the combination of a quasi 2-dimensional MBE grown metal film and an ordered selfassembled overlayer of adsorbed molecules. The molecules can self-assemble to form quasi 1-dimensional conducting columns, in which electrons can be temporarily trapped. Given the short mean free path of conducting electrons, due to surface and impurity scattering, the long range transport is modelled using a diffusion scheme through a discrete lattice, whose sites are defined by the locations of the self-organised molecular columns. Local conductivities are computed using the Kubo formalism and mapped into effective transfer rates between adjacent sites of the lattice. This model includes stochastic excursions of electrons into the molecular states, as well as the quantum mechanical details of short range transport. External nano-engineered gate fields can be used to control the residence time of carriers in molecular bands. Calculations demonstrate that the in-plane conductivity is decreased at low frequency due to electron capture in the molecular columns, and that this effect becomes stronger as the length of the columns is increased.
\end{abstract}

Keywords: Thin metals; Conductivity; Self-organisation; Nanotechnology

\section{INTRODUCTION}

Recent advances in nanoscale imaging and characterisation technology have catalysed studies of the interaction of organic molecules with inorganic surfaces. One such example involves the evaporation of organic molecules, under UHV conditions, onto the surface of an MBE grown ultra-thin $\mathrm{Au}$ film, resulting in a multilayered hybrid system [1]. In well-ordered interfaces, the adsorbed molecules induce a new band structure in the metal, which changes its transport and optical properties. The resulting metal/organic hybrid is potentially sensitive to external perturbations, since the induced band structure is sensitive to changes in the interactions between the metal and the adsorbate molecules. The organic molecules are HAT-6 or Phthalocyanines, both of which are 'discotic' liquid crystals [2]. Owing to their disk-like shape, these molecules self-assemble to form 2-dimensional

\footnotetext{
*Corresponding author. Tel.: +44 113233 4996, Fax: +44 113233 4652, e-mail: pecchia@chem.leeds.ac.uk
} 


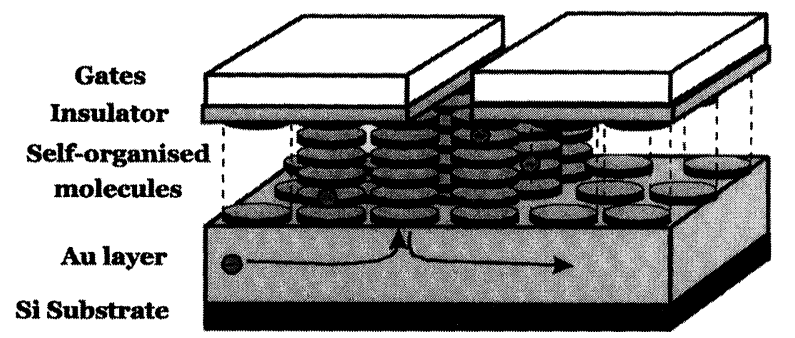

FIGURE 1 The hybrid metal/organic system, in which discotic molecules self-organise on an ultra-thin Au film. The gates enhances the penetration of the metal electrons into the molecular columns.

hexagonal ordered columns. The overlap between the $\pi$ orbitals of the aromatic cores allows electron or hole transport along the columns [3]. The molecules also have hydrocarbon chains, which prevent conduction in the direction perpendicular to the columns, leading to a strong anisotropy in the carrier mobility.

A prototype device that we plan to realise is represented in Figure 1. Metal electrons can be temporarily trapped in the molecular states. The electronic penetration into the organic overlayer and the residence time of the carrier in the molecules can be manipulated by electric gate fields applied along the column axes, as shown in Figure 1. We investigate the consequences of the charge trapping into the columns by calculating the frequency dependent conductivity of the hybrid system.

\section{THE MODEL}

In order to calculate the conductivity of the thin metal film, taking into account the effect of stochastic excursions into the adsorbed molecular columns, we have devised a lattice diffusion model. The very short mean free path encountered in this system, due to surface roughness and molecular scattering, justifies this approach. The metal film is subdivided into a lattice of sites or regions. The lattice comprises two kind of regions: those below the molecular cores, denoted $\mathrm{A}$, and those below the alkali side chains, denoted $\mathbf{B}$, as shown in
Figure 2. The subdivision is based on different behaviour that electrons have in the two regions. This approach enables inclusion of local transport properties, which are determined by quantum interactions. Local conductivities are calculated using the Kubo formula. The calculation includes the effect of quantum confinement and the scattering lifetimes from surface roughness, impurities and phonons [4-6]. In these calculations the metal states are assumed to be plane waves along the metal film and quantum-well states in the direction perpendicular to the film. Surface roughness is included as a perturbing potential. The different contributions to the total resistivity are shown in Figure 3. The resistivity of the film increases sharply for thicknesses below $30 \AA$, when surface roughness scattering dominates over phonon and impurity scattering. The resistivity also shows oscillations, which are a consequence of the sub-band structure of the electronic energy levels.

Local conductivities are mapped into transfer rates between adjacent sites of the lattice. We denote $W_{\mathrm{AB}}$ the transfer rate between a region $\mathrm{A}$ and a region $\mathrm{B}$ of the metal, and $W_{B B}$ the transfer rate between adjacent $\mathrm{B}$ regions. The determination of these rates is realised as follows. When the conductivity of the uncovered metal is considered, there is no actual distinction between $\mathrm{A}$ and $\mathrm{B}$

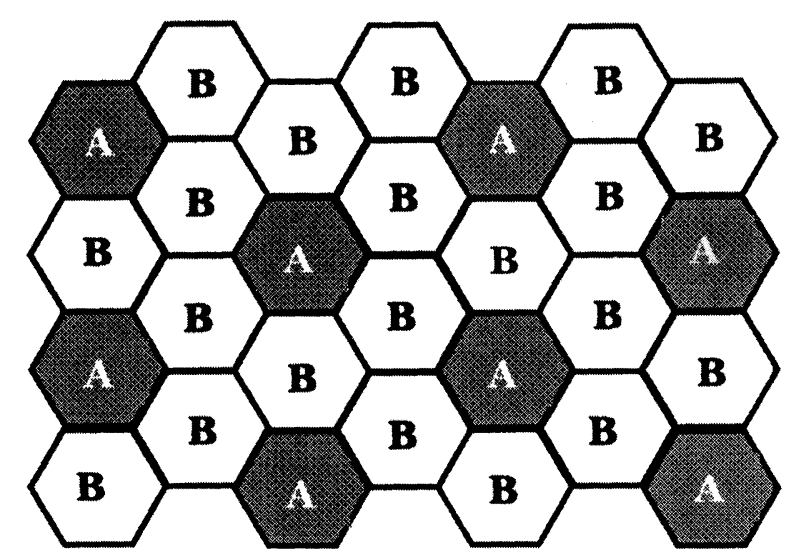

FIGURE 2 Subdivision of the metal film into region ' $A$ ' and ' $\mathrm{B}$ '. The subdivision matches the position of the aromatic cores of the molecules located above the sites A, and the hydrocarbon chains located above the sites B. 


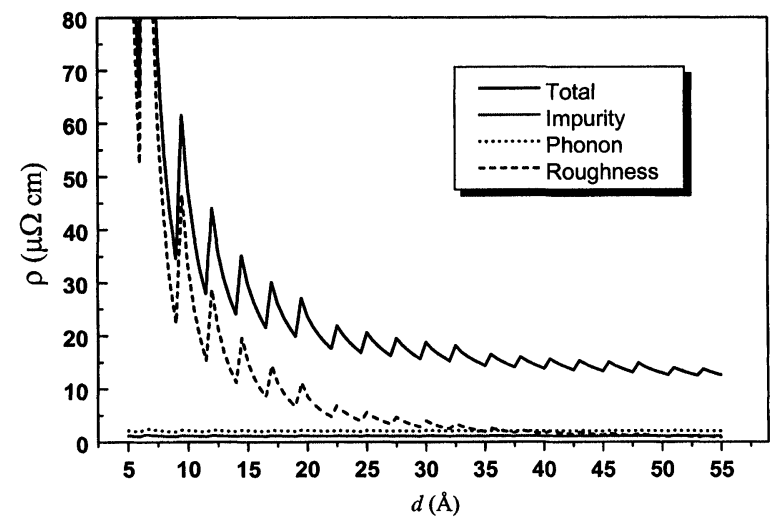

FIGURE 3 Thickness dependent resistivity of a Au film. The different contributions to the scattering from impurity, phonons and surface roughness are shown.

sites. Comparing the conductivity obtained from diffusion theory with the conductivity calculated using the Kubo formula, an effective transfer rate, $W_{\mathrm{BB}}^{(O)}$, can be obtained. When the molecules are present the degeneracy between $\mathrm{A}$ and $\mathrm{B}$ sites is removed and a differentiation between the transfer rates $W_{\mathrm{AB}}$ and $W_{B B}$ is made. The effect of the molecules is simulated by lowering the rate $W_{B B}$ with respect to the unperturbed rate $W_{\mathrm{BB}}^{(O)}$, because of increased scattering induced by the adsorbed hydrocarbon side chains. The rate $W_{\mathrm{AB}}$ is further decreased with respect to the rate $W_{B B}$ to account quantum interactions with the molecular cores. Using a tight-binding calculation, we have estimated for $H A T-6$ on $\mathrm{Au}$, a ratio $W_{\mathrm{AB}} / W_{B B} \approx 0.8$. These effects decrease the conductivity of the metal by an estimated $10 \%$ when the molecules are evaporated.

Real capture of electrons from the metal into the molecules is an activated, phonon assisted, process which has to be treated separately, as a stochastic phenomenon. Electronic trapping into the molecular states occurs mainly from the regions below the cores, where the interaction between metal and molecules is stronger. This enforces the distinction between A and B sites. The capture process is simulated by allowing a transfer from each region A into the closest molecule. In the same way, stochastic transport along the columns can be modelled allowing transfer rates between adjacent molecules. A release rate back into the channel is also included.

Long range conductivity is then calculated from the Master equation of diffusion. This model enables calculation of the frequency response of the conductivity, which is due to the time delay caused by charge trapping into the molecular states. The capture rate into the lowest molecule is assumed to be of the form:

$$
W_{01}=\nu_{1} \exp \left[-\beta\left(\Delta E_{1}-e E_{g} a+e E_{I F} a\right)\right]
$$

where $\Delta E_{1}$ is the difference between the Fermi energy of the metal and the energy of the first empty molecular state, $a$ is the average distance between the metal surface and the centre of mass of the lowest molecular core, $E_{g}$ is the field due to an external gate contact, as shown in Figure 1, and $E_{I F}$ is the total image field due to trapped charges. Since the number of trapped charges depends on the trapping and release rates, and these depend on the image field, the latter has to be calculated self-consistently [1]. The prefactor $\nu_{1}$ accounts for the exact coupling between the electrons and the phonon modes which are responsible for the thermal excitation of electrons into the molecular states [7]. Similarly, it is possible to define a hopping rate from the lowest molecule to the next one in the stack. This rate has a corresponding activation energy $\Delta E_{2}$. The activation energies between the molecules are due to the image charge energy correction. The charged molecular levels have different energies depending on their distance from the metal surface, and the energy differences vanish far from the metal surface. The release rate back into the metal is limited by the rate of phonon emission, which is of the order of $10^{12} \mathrm{~Hz}$. The molecule to molecule transfer rate within a column was estimated using the measured time of flight mobility $\left(\mu \approx 10^{-3} \mathrm{~V}^{-1} \mathrm{~cm}^{2} \mathrm{~s}^{-1}\right)$ [8], which gives an effective rate of $10^{10} \mathrm{~Hz}$. Trapped electrons and interfacial charge transfer contribute to Coulomb scattering. This effect has not yet been analysed. 


\section{RESULTS AND COMMENTS}

Using the model described, we have calculated the frequency dependent conductivity of the hybrid system. Figure 4 shows the conductivity as a function of frequency for different choices of the activation energy between the Fermi energy and the first molecules in the column $\left(\Delta E_{1}\right)$ and between the first and the second molecule $\left(\Delta E_{2}\right)$. For simplicity, it is assumed that the energy difference between the subsequent molecules in the column is zero. The gate field is assumed to be $\mathrm{E}_{\mathrm{g}}=10^{6} \mathrm{~V} / \mathrm{cm}$. The activation energy $\Delta E_{2}$ is assumed to be constant $(0.3 \mathrm{eV})$, whilst $\left(\Delta E_{1}\right)$ is varied between $0.3 \mathrm{eV}$ to $0.5 \mathrm{eV}$. The $\mathrm{DC}$ conductivity decreases when the activation energy is decreased because capture is more favourable. The conductivity rises in correspondence to a frequency related to the capture frequency into the first molecule. As the activation energy increases the capture rate decreases and consequently the critical frequency also decreases. This result could be used to characterise the properties of the interface. At a higher frequency, which in this case is between $10^{9}$ and $10^{10} \mathrm{~Hz}$, there is a second rise in conductivity. This ladder structure is a consequence of the trapping into the next molecule in the stack and depends on the energy difference

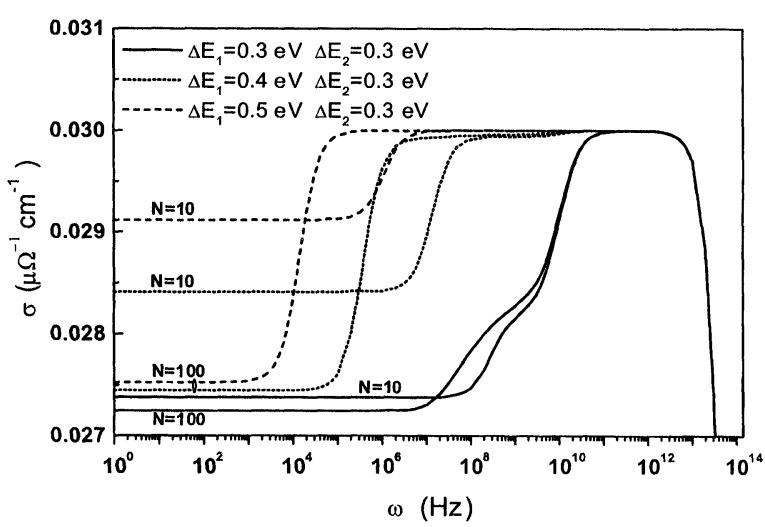

FIGURE 4 Frequency dependent conductivity for a Au film of $20 \AA$ thickness. For each choice of the activation energies $\Delta E_{1}$ and $\Delta E_{2}$, the calculations are performed in the cases of $\mathrm{N}=10$ and $\mathrm{N}=100$ molecular layers.

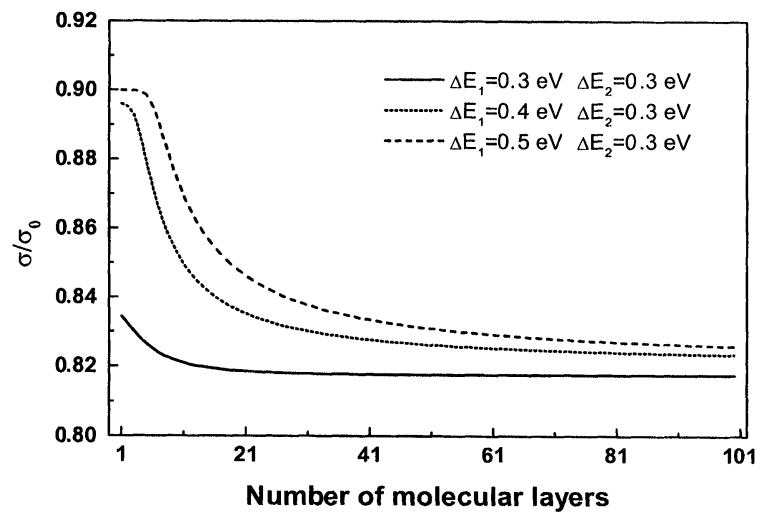

FIGURE 5 DC conductivity normalised to the bare metal film conductivity, as a function of molecular column length, for different activation energies $\Delta E_{1}$ and $\Delta E_{2}$.

$\Delta E_{2}$. For frequencies higher than the plasma frequency the conductivity is Drude-like and drops to zero. These calculations were performed for 10 and 100 molecular layers, after which no significant changes are observed. Figure 5 shows the DC conductivity normalised to the bare metal conductivity, as a function of the thickness of the organic overlayer. The conductivity decreases as more molecular layers are added, because more states are available for the trapped electrons. With this particular choice of parameters, the effect saturates after 100 layers.

\section{CONCLUSIONS}

A model based on diffusion has been developed for calculating long range transport in a thin metal covered by ordered arrays of organic molecules. Calculations show how the frequency dependent conductivity is affected by carrier capture into molecular states. This exhibits a ladder-like structure, which reflects the energy levels of the molecules along the columns.

\section{References}

[1] Pecchia, A. et al. (2000). Microelectronics Engineering, 51(2), 633.

[2] Chandrasekhar, S. and Ranganath, G. S. (1990). Rep. Prog. Phys., 53, 57. 
[3] Boden, N. et al. (1995). Physical Review B, 52(18), 13274.

[4] Trivedi, N. and Ashcroft, N. W. (1988). Physical Review B, 38(17), 12298.
[5] Calecki, D. (1985). J. Phys. C: Solid State Phys., 19, 4315.

[6] Calecki, D. (1990). Physical Review B, 42(11), 6906.

[7] Emin, D. (1975). Advances in Physics, 24, 305.

[8] Adam, D. et al. (1994). Nature, 371, 141. 

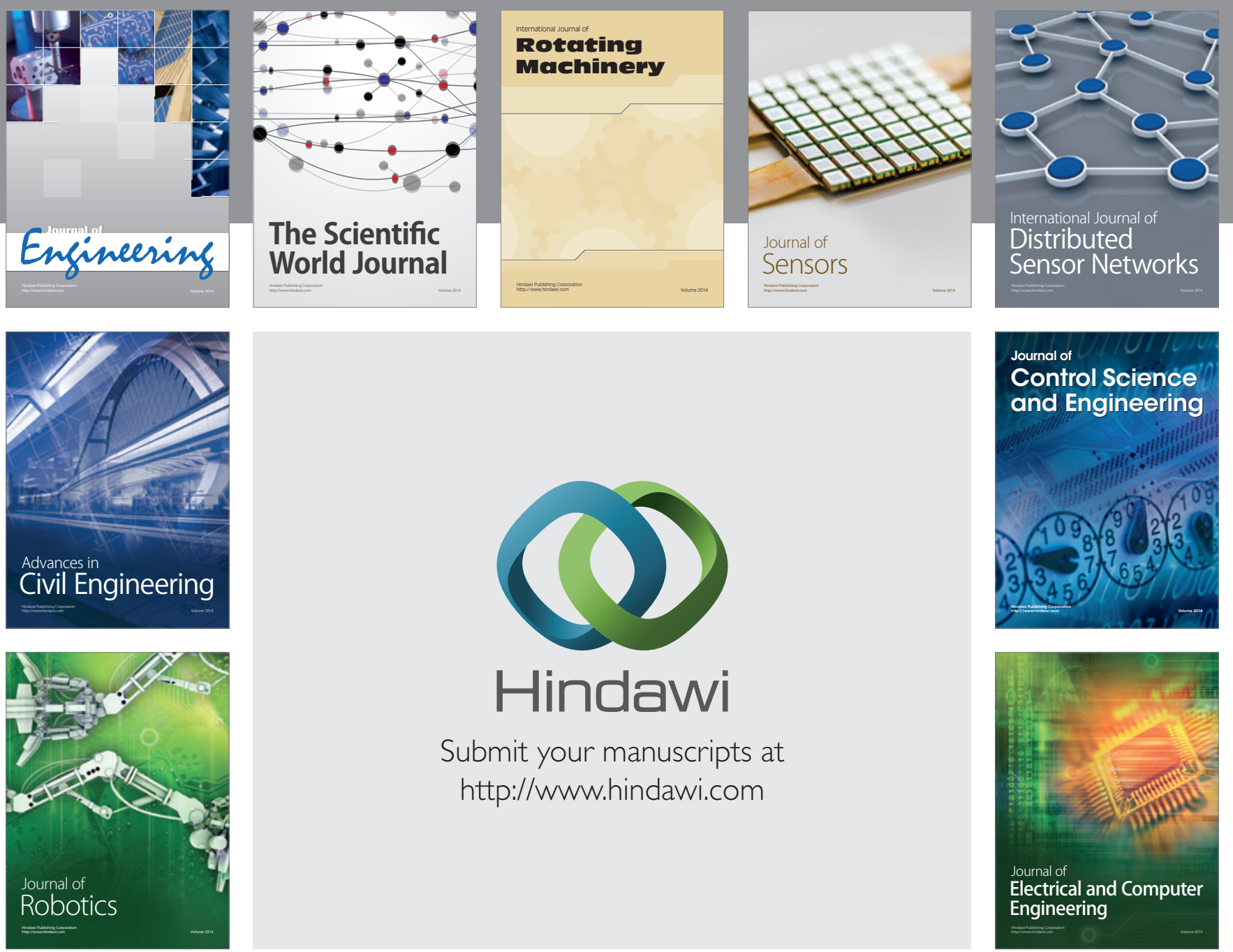

Submit your manuscripts at

http://www.hindawi.com
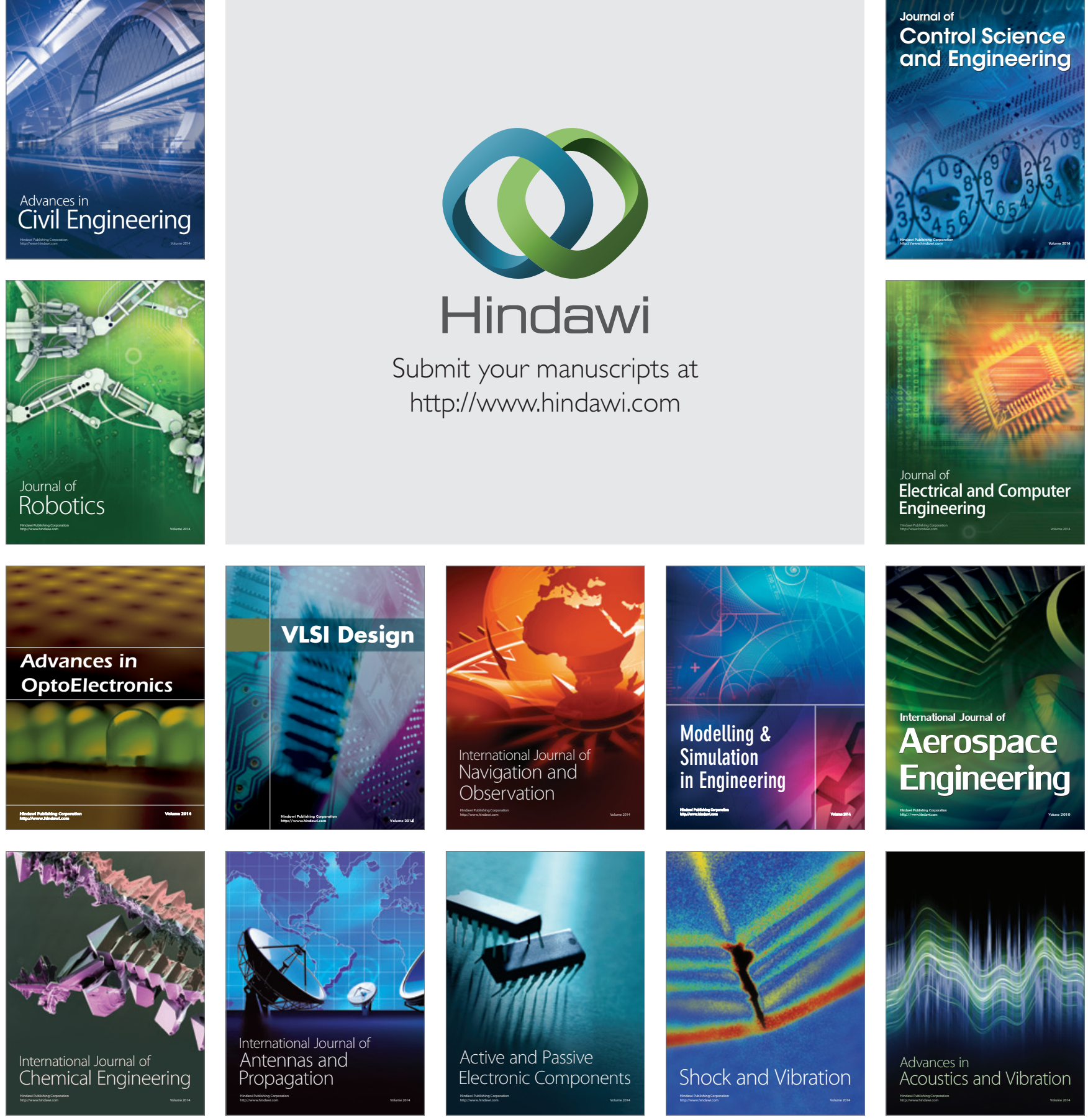